Pacific Journal of Mathematics

SET APPROXIMATION BY LEMNISCATES AND THE
SPECTRUM OF AN OPERATOR ON AN INTERPOLATIOn
SPACE

JaMES D. STAFNeY 


\title{
SET APPROXIMATION BY LEMNISCATES AND THE SPECTRUM OF AN OPERATOR ON AN INTERPOLATION SPACE
}

\author{
JAMES D. STAFNeY
}

Let $B_{0}, B_{1}$ be an interpolation pair of Banach spaces and $T_{s}$ a bounded linear operator on the corresponding interpolation space $\left[B_{0}, B_{1}\right]_{s}, 0 \leqq s \leqq 1$, such that the operators $T_{s}$ all agree on $B_{0} \cap B_{1}$. In this paper we extend our previous work by giving a general upper bound for the spectrum of $T_{s}$ constructed from the spectra of $T_{0}$ and $T_{1}$ using a set interpolation formula which we introduce in $\$ 1$ for compact sets in the plane. In $\$ 3$ we show that this upper bound is essentially best possible. This requires a theorem about approximating sets with lemniscates, which we prove in $\$ 2$. Finally, we show in $\$ 4$ that under certain conditions the operators $T_{s}, 0 \leqq s \leqq 1$, all have the same spectrum.

1. Upper bound for spectrum. In this section we introduce the notion of interpolating between two sets in the complex plane (see 1.3) and then we obtain an upper bound for the spectrum of an operator on an interpolation space by "interpolating its extreme spectra (see 1.9)."

Basic situation 1.1. $B_{0}, B_{1}$ are two Banach spaces continuously embedded in a topological vector space $V$ so that $B_{0} \cap B_{1}$ is dense in both $B_{0}$ and $B_{1}$. The corresponding spaces $\left[B_{0}, B_{1}\right]_{s}, 0 \leqq s \leqq 1$, are the spaces defined in $\left[2\right.$, p. 114]. By $[2, \S 9.3], B_{j}=\left[B_{0}, B_{1}\right]_{j}, j=$ 0,1 . $T_{s}, 0 \leqq s \leqq 1$, is a bounded linear operator on $\left[B_{0}, B_{1}\right]_{s}$ and all the operators $T_{s}$ agree on $B_{0} \cap B_{1}$.

General notation. For a normed linear space $B,\|\|_{B}$ will denote the norm of $B$. We will sometimes use \|\| to denote \|\|$_{B}$ if no confusion results. The space of bounded linear operators on a normed linear space $B$ with the operator norm will be denoted $O(B)$. We will call bounded linear operators, operators. If $T$ is an operator, $\operatorname{sp}(T)$ will denote the spectrum of $T$ and $r(T)$ the spectral radius of $T$, the smallest number $r$ such that $\operatorname{sp}(T) \subset\{z:|z| \leqq r\}$. If $F$ is analytic on $\operatorname{sp}(T)$, then $F(T)$ will denote the operator $(2 \pi i)^{-1} \int_{\gamma}(\lambda I-T)^{-1} d \lambda$ where $\gamma$ is an envelope of $\operatorname{sp}(T)$ in the domain of $F$. If $E$ is a subset of the complex plane, $R(E)$ will denote the rational functions with poles in the complement of $E$. The following is proved in [2]. 
LEMMA 1.2. If $T_{s}, 0 \leqq s \leqq 1$, is as in 1.1 then

$$
\left\|T_{s}\right\| \leqq\left\|T_{0}\right\|^{1-s}\left\|T_{1}\right\|^{s}
$$

If $B_{0}$ and $B_{1}$ are as in 1.1 and $T_{j}$ is an operator on $B_{j}, j=0,1$, such that $T_{0}$ and $T_{1}$ agree on $B_{0} \cap B_{1}$, then there is an operator $T_{s}$ on $\left[B_{0}, B_{1}\right]_{s}, 0 \leqq s \leqq 1$, such that all the operators $T_{s}$ agree on $B_{0} \cap B_{1}$.

We next introduce the notion of interpolation sets $I_{s}\left(E_{0}, E_{1}\right)$ corresponding to two given compact sets $E_{0}, E_{1}$ in the plane. These interpolation sets will be used to provide an upper bound for the spectrum of operators $T_{s}$.

If $F$ is a complex valued function and $t$ is a positive real number, let $L(t, F)$ denote the set of elements $x$ in the domain of $F$ such that $|F(x)| \leqq t$.

Definition 1.3 . Let $E_{0}, E_{1}$ be compact nonempty sets in the plane such that $E_{0} \subset E_{1}$. For $0 \leqq s \leqq 1$ we define the set $I_{s}\left(E_{0}, E_{1}\right)$ to be $\cap L\left(r^{s}, F\right)$ where the intersection is taken over all pairs $(r, F)$ such that: (a) $r$ is a real number $>1$, (b) $F$ is an analytic function on an open set in the plane, (c) $L(r, F)$ is a compact subset of the domain of $F$ and (d) $E_{j}$ is a subset of the interior of $L\left(r^{j}, F\right), j=0,1$.

The following lemma is an immediate consequence of the preceding definition.

LEMMA 1.4. Suppose that $E_{0}, E_{1}, F_{0}$ and $F_{1}$ are compact subsets of the plane and that $E_{0} \subset E_{1}, F_{0} \subset F_{1}, E_{0} \subset F_{0}$ and $E_{1} \subset F_{1}$. Then,

$$
I_{s}\left(E_{0}, E_{1}\right) \subset I_{s}\left(F_{0}, F_{1}\right) \quad 0 \leqq s \leqq 1 \text {. }
$$

For a complex number $z$ and a positive real number $t$ let $D(z, t)$ denote the set of complex numbers $w$ such that $|z-w|<t$. Let $D(z, t]$ denote the closure of $D(z, t)$. For a set $A$ in a topological space int $A$ will denote the interior of $A$.

LEMMA 1.5. Let $K$ be a compact subset of $D(0,1)$. To each $\epsilon>0$ and $s, 0<s<1$, there corresponds a $\delta>0$ such that for each $\zeta$ in $K, D(\zeta, \delta] \subset D(0,1)$ and

$$
I_{s}(D(\zeta, \delta], D(0,1]) \subset K+D(0, \epsilon) .
$$

Proof. For $\zeta$ in $K$, let $\lambda_{\zeta}$ denote the analytic function defined by $\lambda_{\zeta}(z)=(z-\zeta) /(1-z \bar{\zeta})$ on the complement of $\left\{\bar{\zeta}^{-1}\right\}$. Choose $u$ such that 
$1<u<|1 / \zeta|$ for each $\zeta$ in $K$. Since $K$ is compact in $D(0,1)$ it is clear that

(1) corresponding to $K, \epsilon, s$ there is an $r>1$ such that if $\zeta \in K$ and $\left|(r / u) \lambda_{\zeta}(z)\right| \leqq r^{s}$, then $|z-\zeta|<\epsilon$.

It is clear that we can choose $\delta$ so that $D(\zeta, \delta] \subset \operatorname{int} L\left(1,(r / u) \lambda_{\zeta}\right)$ and $D(\zeta, \delta] \subset D(0,1)$ for each $\zeta$ in $K$. Let $\zeta$ be in $K$. Let $F(z)=$ $(r / u) \lambda_{\zeta}(z)$. From what we have observed and Definition 1.3 it follows that

$$
I_{s}\left(D(\zeta, \delta], D(0,1] \subset L\left(r^{s}, F\right)\right.
$$

It follows from (1) that $L\left(r^{s}, F\right) \subset K+D(0, \epsilon)$; and, this together with (2) proves the lemma.

LeMmA 1.6. Let $B_{0}, B_{1}$ and $T_{s}, 0 \leqq s \leqq 1$, be as in 1.1. Suppose that $E$ is a compact set in the plane such that $\operatorname{sp}\left(T_{0}\right), \operatorname{sp}\left(T_{1}\right) \subset E$ and for each $\lambda \notin E,\left(\lambda-T_{0}\right)^{-1}$ and $\left(\lambda-T_{1}\right)^{-1}$ agree on $B_{0} \cap B_{1}$. Then $\operatorname{sp}\left(T_{s}\right) \subset E$, $0 \leqq s \leqq 1$; and, if $F$ is analytic on $E$, then the operators $F\left(T_{s}\right)$ all agree on $B_{0} \cap B_{1}$.

Proof. Fix an $s$ in the interval $[0,1]$. We will first show that

$$
\operatorname{sp}\left(T_{s}\right) \subset E \text {. }
$$

If $\lambda \notin E$, then by the restrictions on $E$ the operators $\left(\lambda-T_{0}\right)^{-1}$ and $\left(\lambda-T_{1}\right)^{-1}$ agree on the space $B_{0} \cap B_{1}$. From 1.2 it follows that there is an operator $Q$ on $B_{s}$ such that $Q$ and $\left(\lambda-T_{0}\right)^{-1}$ agree on $B_{0} \cap B_{1}$; in particular, $Q\left(\lambda-T_{s}\right)$ and $\left(\lambda-T_{s}\right) Q$ are equal to the identity on $B_{0} \cap$ $B_{1}$. Since both $Q$ and $T_{s}$ are bounded on $B_{s}$, we see that $Q$ is the inverse of $\lambda-T_{s}$; hence, $\lambda \notin \mathrm{sp}\left(T_{s}\right)$. Our argument establishes (3).

We next observe that if $\lambda \notin E$, then the operators $\left(\lambda-T_{s}\right)^{-1}, 0 \leqq s \leqq$ 1 , and agree on $B_{0} \cap B_{1}$. To see this we first note that since $\left(\lambda-T_{0}\right)^{-1}$ and $\left(\lambda-T_{1}\right)^{-1}$ agree on $B_{0} \cap B_{1}$, these two operators leave $B_{0} \cap B_{1}$ invariant. If $y \in B_{0} \cap B_{1}$, then $y=\left(\lambda-T_{0}\right) x$ where $x=\left(\lambda-T_{0}\right)^{-1} y$, which is an element in $B_{0} \cap B_{1}$. Thus,

$$
\left(\lambda-T_{s}\right)^{-1} y=\left(\lambda-T_{s}\right)^{-1}\left(\lambda-T_{0}\right) x=\left(\lambda-T_{s}\right)^{-1}\left(\lambda-T_{s}\right) x=x .
$$

Since $y$ was chosen arbitrarily in $B_{0} \cap B_{1}$ and $x$ does not depend on $s$, we have shown that the operators $\left(\lambda-T_{s}\right), 0 \leqq s \leqq 1$, all agree on $B_{0} \cap B_{1}$. Consequently, the operators $G\left(T_{s}\right), 0 \leqq s \leqq 1$, all agree on $B_{0} \cap B_{1}$ if $G \in R(E)$. Let $G_{n}$ be a sequence of elements in $R(E)$ such 
that $G_{n}$ converges uniformly to $F$ on some neighborhood of $E$. Then $G_{n}\left(T_{0}\right)$ converges to $F\left(T_{0}\right)$ in $O\left(B_{0}\right)$ and $G_{n}\left(T_{1}\right)$ converges to $F\left(T_{1}\right)$ in $O\left(B_{1}\right)$. If $x \in B_{0} \cap B_{1}$, then $G_{n}\left(T_{0}\right) x=G_{n}\left(T_{1}\right) x$ for $n=1,2, \cdots$. Since $G_{n}\left(T_{0}\right) x$ converges to $F\left(T_{0}\right) x$ in $B_{0}$ and $F\left(T_{1}\right) x$ in $B_{1}$ and since $B_{0}$ and $B_{1}$ are continuously embedded in $V$, it follows that $F\left(T_{0}\right) x=F\left(T_{1}\right) x$. This completes the proof.

Comment. In view of Lemma 1.6 it is natural to ask whether the set $E$ in 1.6 can always be taken to be $\operatorname{sp}\left(T_{0}\right) \cup \operatorname{sp}\left(T_{1}\right)$. We have not been able to answer this question. The best that we have done is the content of the next lemma.

LEMMA 1.7. Let $B_{0}, B_{1}, T_{0}$ and $T_{1}$ be as in 1.1. Then the set $H=\left\{\lambda: \lambda \notin \mathrm{sp}\left(T_{0}\right) \cup \mathrm{sp}\left(T_{1}\right)\right.$ and $\left(\lambda-T_{0}\right)^{-1},\left(\lambda-T_{1}\right)^{-1}$ agree on $\left.B_{0} \cap B_{1}\right\}$ contains the unbounded component of the complement of $\operatorname{sp}\left(T_{0}\right) \cup \operatorname{sp}\left(T_{1}\right)$; and, the set $H$ is a union of components of the complement of $\operatorname{sp}\left(T_{0}\right) \cup$ $\operatorname{sp}\left(T_{1}\right)$.

Proof. Suppose $\lambda$ is in the unbounded component of the complement of $E=\operatorname{sp}\left(T_{0}\right) \cup \operatorname{sp}\left(T_{1}\right)$. There is a sequence $p_{n}$ of polynomials which converges uniformly to $(\lambda-z)^{-1}$ for $z$ in a neighborhood of $E$. It follows that $p_{n}\left(T_{j}\right)$ converges to $\left(\lambda-T_{j}\right)^{-1}$ in the operator norm of $B_{j}$, $j=0,1$. If $x \in B_{0} \cap B_{1}$, then $p_{n}\left(T_{j}\right) x$ converges to $\left(\lambda-T_{j}\right)^{-1} x$ in $B_{j}$. Therefore, $p_{n}\left(T_{j}\right) x$ converges to $\left(\lambda-T_{j}\right)^{-1} x$ in the topology of $V$, $j=0,1$. Since $T_{0}$ and $T_{1}$ agree on $B_{0} \cap B_{1}, p_{n}\left(T_{0}\right) x=p_{n}\left(T_{1}\right) x$ for each $n$. Thus, $\left(\lambda-T_{0}\right)^{-1} x=\left(\lambda-T_{1}\right)^{-1} x$. If $\lambda$ is a point in some bounded component $V$ of the complement of $E$ such that $\left(\lambda-T_{0}\right)^{-1}$ and $\left(\lambda-T_{1}\right)^{-1}$ agree on $B_{0} \cap B_{1}$ and if $\lambda_{1}$ is also in $V$, then there is a sequence of polynomials $p_{n}(n=1,2, \cdots)$ such that $p_{n}(1 /(\lambda-z))$ converges uniformly to $1 /\left(\lambda_{1}-z\right)$ for $z$ in some neighborhood of $E$. The same reasoning as above shows that $\left(\lambda_{1}-T_{0}\right)^{-1}$ and $\left(\lambda_{1}-T_{1}\right)^{-1}$ agree on $B_{0} \cap B_{1}$. This completes the proof.

LeMMA 1.8. Let $B_{0}, B_{1}$ and $T_{s}, 0 \leqq s \leqq 1$, be as in 1.1. Then

$$
r\left(T_{s}\right) \leqq r\left(T_{0}\right)^{1-s} r\left(T_{1}\right)^{s}, \quad 0 \leqq s \leqq 1
$$

Proof. If $\epsilon>0$, then there corresponds on $n_{0}$ such that

$$
\left\|\left(T_{j}\right)^{n}\right\| \leqq\left(r\left(T_{j}\right)+\epsilon\right)^{n} \quad j=0,1 ; n \geqq n_{0} .
$$

Since the operators $\left(T_{s}\right)^{n}, 0 \leqq s \leqq 1$, all agree on $B_{0} \cap B_{1}$, it follows from 1.2 that 


$$
\left\|\left(T_{s}\right)^{n}\right\| \leqq\left\|\left(T_{1}\right)^{n}\right\|^{s}, \quad 0 \leqq s \leqq 1 ; n=1,2, \cdots
$$

Since $\epsilon$ was chosen arbitrarily, the conclusion of the lemma follows from our observations and the fact that $r\left(T_{s}\right)=\lim \left\|\left(T_{s}\right)^{n}\right\|^{1 / n}$.

THEOREM 1.9. Suppose that $B_{0}, B_{1}$ and $T_{s}, 0 \leqq s \leqq 1$, are as in 1.1 and that $\operatorname{sp}\left(T_{0}\right) \subset \operatorname{sp}\left(T_{1}\right)$. Let $E$ denote the complement of $\left\{\lambda: \lambda \notin \operatorname{sp}\left(T_{1}\right)\right.$ and $\left(\lambda-T_{0}\right)^{-1},\left(\lambda-T_{1}\right)^{-1}$ agree on $\left.B_{0} \cap B_{1}\right\}$. Then

$$
\operatorname{sp}\left(T_{s}\right) \subset I_{s}\left(\operatorname{sp}\left(T_{0}\right), E\right)
$$

Proof. Suppose that the theorem is false; that is, suppose that there is a $\lambda$ in $\operatorname{sp}\left(T_{s}\right)$ which is not in $I_{s}\left(\operatorname{sp}\left(T_{0}\right), E\right)$. In particular, there is a pair $(r, F)$ corresponding to the sets $\operatorname{sp}\left(T_{0}\right)$ and $E$ as in 1.3 such that $\lambda \notin L\left(r^{s}, F\right)$. However, since $E \subset$ domain of $F$ and 1.6 implies that $\lambda \in E$, we conclude that

$$
|F(\lambda)|>r^{s}
$$

Define the operators $U_{t}=F\left(T_{t}\right), 0 \leqq t \leqq 1$, in the usual manner of spectral theory. Since $\lambda \in \operatorname{sp}\left(T_{s}\right)$ it follows from the spectral mapping theorem that

$$
F(\lambda) \in \operatorname{sp}\left(U_{s}\right)
$$

From 1.6 it follows that the operators $U_{t}, 0 \leqq t \leqq 1$, all agree on $B_{0} \cap B_{1}$; consequently, we conclude from 1.8 that

$$
r\left(U_{s}\right) \leqq r\left(U_{0}\right)^{1-s} r\left(U_{1}\right)^{s}
$$

By the choice of $F, \operatorname{sp}\left(T_{j}\right) \subset\left\{z:|F(z)| \leqq r^{j}\right\}, j=0,1$. From this and the spectral mapping theorem we obtain

$$
\operatorname{sp}\left(U_{j}\right) \subset\left\{z:|z| \leqq r^{i}\right\}, \quad j=0,1 .
$$

If we combine (5) thru (7) we get $|F(\lambda)| \leqq r^{s}$, which contradicts (4). This completes the proof.

2. Set approximation by lemniscates. The main purpose of this section is the proof of the approximation theorem, Theorem 2.6.

By a simple set we mean a compact set with dense interior and with a boundary consisting of a finite number of disjoint simple closed curves which are also regular analytic curves (see $[1$, p. 226]). For notation we 
use $\partial E$ and int $E$ to denote the boundary and the interior, respectively, of a set $E$ in the plane.

REMARK 2.1. By a harmonic triple in this paper we mean what is defined in $[4,5.1]$ except that we do not require that the interior of $E_{1}$ be connected. The main result about harmonic triples that we will need is $[4,5.3]$; and this result is still valid because it can be applied to the closure of each component of the interior of $E_{1}$.

LEMMA 2.2. Let $E_{0}$ and $E_{1}$ be compact subsets of the plane such that $E_{0} \subset E_{1}$ and each component of the complement of $E_{0}$ intersects the complement of $E_{1}$. To each $\epsilon>0$ there corresponds a harmonic triple $\left(D_{0}, D_{1}, \omega\right)$ such that: (a) $D_{0}$ and $D_{1}$ are simple sets and (b) $E_{j} \subset$ int $D_{j} \subset$ $E_{j}+D(0, \epsilon), j=0,1$.

In order to prove this lemma we will use the following topological lemma.

LEMMA 2.3. Let $E_{0}$ and $E_{1}$ be sets which satisfy the conditions of Lemma 2.2. To each $\epsilon>0$ there corresponds a pair of sets $D_{0}, D_{1}$ which satisfy (a) and (b) of Lemma 2.2, $D_{0} \subset$ int $D_{1}$ and each component of the complement of $D_{0}$ intersects the complement of $D_{1}$.

Given sets $E_{0}, E_{1}$ as in Lemma 2.1 let $D_{0}, D_{1}$ be the sets corresponding to $\epsilon$ in Lemma 2.3. To complete the proof of Lemma 2.2 we need only show that there is an $\omega$ such that $\left(D_{0}, D_{1}, \omega\right)$ is a harmonic triple. Let $U$ be a component of the set int $D_{1} \mid D_{0}$. The set $U$ is a subset of a component $V$ of the complement of $D_{0}$. If $\partial U \subset D_{0}$, then $\partial U \subset \partial V$; however, this would imply that $U=V$, since $V$ is connected. But, $V \neq U$ because $V$ contains a point in the complement of $D_{1}$. Let $x$ be a point in $\partial U$ that is not in $D_{0}$. In particular, $x \in V$. Since $U$ is a component of int $D_{1} \mid D_{0}$, it follows that $x$ is not in int $D_{1}$. Thus $x \in \partial D_{1}$. The boundary of $U$ is the union of two disjoint subsets $A$ and $B$ where $A \subset \partial D_{0}$ and $B \subset \partial D_{1}$. We have argued above that $B$ is nonempty; however, it is possible that $A=\varnothing$. If $y$ is in $A$ or $B$, then $y$ must be contained in an arc that lies in $A$ or $B$ since $U$ is a component of int $D_{1} \mid D_{0}$. It follows from the general theory on the Dirichlet problem that there is a continuous function $\omega_{0}$ on the closure of $U$ which is identically 1 on $B$, identically 0 on $A$ and harmonic on $U$. We will now define $\omega$ on $D_{1}$ : Let $\omega$ be defined on the closure of each component of int $D_{1} \mid D_{0}$ in the same way that $\omega_{0}$ was defined on the closure of $U$ and define $\omega$ on $D_{0}$ to be identically 0 . We claim that $\left(D_{0}, D_{1}, \omega\right)$ is harmonic triple (see 2.1); in fact, this is clear except, possibly for (iii) of $[4,5.1]$. Suppose $x \in D_{1} \mid D_{0}$. If $x \in \partial D_{1}$, then 
$\omega(x)=1$. Since each component of int $D_{1} \backslash D_{0}$ has a boundary point in $\partial D_{1}$ (We showed this above.) It follows from the minimum principle that $\omega$ does not assume the value 0 on int $D_{1} \mid D_{0}$. These observations verify that condition (iii) of $[4,5.1]$ is met.

Lemma 2.4. Suppose that $\left(D_{0}, D_{1}, \omega\right)$ is a harmonic triple and that $D_{0}, D_{1}$ are simple sets. Then to each $\epsilon, \eta>0$, there corresponds an $f$ in $R\left(D_{1}\right)$ and a positive real number $b$ such that

$$
|\omega(z)-b \log | f(z)||<\epsilon, \quad \text { if } \quad \omega(z) \geqq \eta .
$$

Proof. Because of Runge's theorem it clearly suffices to prove Lemma 2.4 for the case where $D_{1}$ is a connected simple set, so we will assume that $D_{1}$ is a connected simple set. Let $\omega_{n}, n=1,2, \cdots$, be the function defined on the domain of $\omega$ as follows: $\omega_{n}(z)=1 / n$ if $\omega(z) \leqq 1 / n$ and $\omega_{n}(z)=\omega(z)$ if $\omega(z)>1 / n$. Locally, each $\omega_{n}$ is either a harmonic function or the maximum of two harmonic functions. Thus, each $\omega_{n}$ is subharmonic; and, since the sequence $\omega_{n}$ decreases pointwise to $\omega$, we see that $\omega$ is also subharmonic. Let $U$ denote the interior of $E_{1}$. Since $\omega$ is subharmonic on the domain $U$, the Riesz theorem $[5$, p. 48] implies that

$$
\omega(z)=u(z)+\int \log |z-a| d \mu(a), \quad z \in U
$$

where $u$ is harmonic on $U$ and $\mu$ is a positive Borel measure, supported on $D_{0}$. One can easily show that there is a positive integer $n$ and a finite number of points $a$, in $D_{0}$ such that

$$
\text { (2) }\left|\int_{D_{0}} \log \right| z-a\left|d \mu(a)-n^{-1} \sum_{i} \log \right| z-a_{j}||<\epsilon, \quad \text { for } \quad \omega(z) \geqq \eta \text {. }
$$

We will now establish the following.

2.5. There is a continuous function $u_{1}$ on $D_{1}$ such that: $u_{1}$ is harmonic on $U,\left|u(z)-u_{1}(z)\right|<\epsilon$ for $z$ in $D_{1}$ and all the periods of the harmonic conjugate of $u_{1}$ are rational multiples of $2 \pi$.

We may assume that the boundary of $D_{1}$ has at least two components. Let $\gamma_{n+1}$ denote the boundary component of $D_{1}$ which also forms the boundary of the unbounded component of the complement of $D_{1}$ and let $\gamma_{1}, \cdots, \gamma_{n}$ denote the other boundary components of $D_{1}$. Let $\omega_{j}$ denote the harmonic measure of $\gamma_{j}, j=1, \cdots, n$, with respect to the region $U\left[1\right.$, p. 245]. Let $a_{k, j}$ denote the period of the harmonic conjugate of $\omega_{j}$ corresponding to the curve $\gamma_{k}$ (see [1, p. 162]). We define $u_{1}$ by: 


$$
u_{1}=u+\Sigma \epsilon_{j} \omega_{j} \quad(j=1,2, \cdots, n)
$$

for an appropriate choice of the numbers $\epsilon_{j}$. Since the matrix $\left(a_{k, j}\right)$, $0 \leqq k, j \leqq n$, is a nonsingular matrix $[1, \mathrm{p} .246]$ and since the period of the harmonic conjugate of $u_{1}$ corresponding to the curve $\gamma_{k}$ is $p_{k}+\Sigma_{j} \epsilon_{j} a_{k, j}$, where $p_{k}$ is the period of the harmonic conjugate of $u$ corresponding to $\gamma_{k}$, it follows that we can choose the numbers $\epsilon_{j}$ in such a way that $-\epsilon<\epsilon_{j}<\epsilon$ for each $j$ and the numbers $p_{k}+\Sigma_{j} \epsilon_{j} a_{k, j}, k=1, \cdots, n$, are all rational multiples of $2_{\pi}$. The conclusion of (2.5) follows from the above remarks and the maximum principle.

We now return to the proof of Lemma 2.4. Since the boundary curves of $D_{1}$ are analytic curves and $\omega$ is constant on each component of the boundary of $D_{1}$ it follows from the reflection principle that $\omega$ has an extension to a function which is harmonic on a neighborhood of $D_{1}$. Since the integral in (1) is clearly harmonic on a neighborhood of the boundary of $D_{1}$, it follows that $u$ has an extension which is harmonic on a neighborhood of $D_{1}$. Consequently, $u_{1}$ also has an extension which is harmonic on a neighborhood of $D_{1}$. By 2.5 we can choose an integer $m$ such that the periods of the harmonic conjugate $h$ of $m u_{1}$ are all multiples of $2_{\pi}$. Let $g=e^{m u_{1}+i h}$. From what we have noted above we conclude that $g$ has an extension which is analytic on a neighborhood of $D_{1}$. Since $g$ has no zeros in $D_{1}$ we can use Runge's theorem to choose an element $g_{1}$ in $R\left(D_{1}\right)$ such that

$$
\left|m^{-1} \log \right| g_{1}(z)\left|-u_{1}(z)\right|<\epsilon, \quad z \in D_{1} .
$$

Let $p(z)=\Pi_{j}\left(z-a_{j}\right)$ where the $a_{j}$ are the numbers that appear in (2). From (1), (2), 2.5, and (3) we obtain

$$
\left|\omega(z)-(m n)^{-1} \log \right| p(z)^{m} g_{1}(z)^{n}||<3 \epsilon, \omega(z) \geqq \eta .
$$

This completes the proof of Lemma 2.4.

THEOREM 2.6. Suppose that $E_{0}, E_{1}$ are two compact sets in the plane, that $E_{0} \subset E_{1}$ and that each component of the complement of $E_{0}$ intersects the complement of $E_{1}$. To each $\epsilon_{1}>0$ there corresponds an $F$ in $R\left(E_{1}\right)$ and an $r>1$ such that

$$
E_{i} \subset \operatorname{int} L\left(r^{i}, F\right) \subset E_{i}+D\left(0, \epsilon_{1}\right), \quad j=0,1 .
$$

Proof. First choose a harmonic triple $\left(D_{0}, D_{1}, \omega\right)$ corresponding to $\epsilon_{1}$ as in Lemma 2.2. In particular,

$$
D_{j} \subset E_{j}+D\left(0, \epsilon_{1}\right), \quad j=0,1 \text {. }
$$


Choose $\epsilon, \eta>0$ so that

$$
\begin{aligned}
& \{z: \omega(z) \leqq 2 \epsilon+\eta\} \subset E_{0}+D\left(0, \epsilon_{1}\right), \\
& \epsilon+\eta<1-\epsilon \text { and } \\
& E_{1} \subset \operatorname{int}\{z: \omega(z) \leqq 1-2 \epsilon\} .
\end{aligned}
$$

Corresponding to $\epsilon$ and $\eta$ we choose $b$ and $f$ as in Lemma 2.4. For convenience, let $h=b \log |f|$. Let $H_{0}=\{z: h(z) \leqq \epsilon+\eta\}$. Since $h$ is subharmonic on $\{z: \omega(z)<\eta\}$ and $h \leqq \epsilon+\eta$ on the set $\{z: \omega(z)=\eta\}$, it follows that $h \leqq \epsilon+\eta$ on $\{z: \omega(z) \leqq \eta\}$. In particular, $D_{0} \subset H_{0}$. Thus,

$$
E_{0} \subset \text { int } H_{0} \text {. }
$$

Since $h$ is superharmonic on the complement of $D_{1}$ and $h \geqq 1-\epsilon$ on $\{z: \omega(z)=1\}$, we have

$$
h(z) \geqq 1-\epsilon, \quad z \notin D_{1} .
$$

Suppose $z \in H_{0}$, so $h(z) \leqq \epsilon+\eta$. Since $\epsilon+\eta<1$ least. Thus either $\omega(z) \leqq \eta$ or $\omega(z) \leqq 2 \epsilon+\eta$. Consequumy, num (v) we obtain

$$
H_{0} \subset E_{0}+D\left(0, \epsilon_{1}\right)
$$

Let $H_{1}=\{z: H(z) \leqq 1-\epsilon\}$. We have already noted that if $\omega(z) \leqq$ $\eta$, then $h(z) \leqq \epsilon+\eta$. From this and (6) it follows that $h(z) \leqq 1-\epsilon$ if $\omega(z) \leqq 1-2 \epsilon$. Thus

$$
H_{1} \supset\{z: \omega(z) \leqq 1-2 \epsilon\} .
$$

We conclude from (7) that

$$
E_{1} \subset \text { int } H_{1} \text {. }
$$

From (9) and the definition of $H_{1}$ we have $H_{1} \subset D_{1}$; consequently,

$$
H_{1} \subset E_{1}+D\left(0, \epsilon_{1}\right)
$$

We define $F$ and $r$ as follows: $F=e^{-(\epsilon+\eta) / b} f$ and $r=$ $e^{[(1-\epsilon)-(\epsilon+\eta)] / b}$. Evidently, $r>1, F \in R\left(E_{1}\right)$ and $H_{j}=L\left(r^{j}, F\right)$ for $j=$ 0,1 . The conclusion of the theorem now follows from (8), (10), (11), and (12). 
3. The upper bound is best possible. The purpose of this section is to establish Theorem 3.1. This theorem gives a class of operators for which the upper bound given in Theorem 1.9 is attained; therefore, it shows, in particular, that an upper bound for $\operatorname{sp}\left(T_{s}\right)$ which depends only on $\operatorname{sp}\left(T_{0}\right)$ and $\operatorname{sp}\left(T_{1}\right)$ can be no better than the one given in Theorem 1.9 provided that $\operatorname{sp}\left(T_{0}\right)$ and $\operatorname{sp}\left(T_{1}\right)$ satisfy the one further condition imposed in Theorem 3.1.

If $E$ is a compact set in the plane, then $C_{R}(E)$ will denote all functions which are the uniform limit of sequences in $R(E)$.

THEOREM 3.1. Suppose that $E_{0}, E_{1}$ are compact subsets of the plane, $E_{0} \subset E_{1}$, the complement of $E_{1}$ meets each component of the complement of $E_{0}$ and that the zero function is the only function in $C_{R}\left(E_{1}\right)$ which vanishes identically on $E_{0}$. Let $B_{k}=C_{k}\left(E_{k}\right), k=0,1, V=B_{0}$ and identify $B_{0}, B_{1}$ with their natural embeddings in $V$. Let $T_{s}$ be the bounded linear operators on $\left[B_{0}, B_{1}\right]_{s}, 0 \leqq s \leqq 1$, such that $T_{s} f(z)=z f(z), z \in E_{0}$, $f \in B_{1}$. Then

$$
\operatorname{sp}\left(T_{s}\right)=I_{s}\left(E_{0}, E_{1}\right), \quad 0 \leqq s \leqq 1
$$

Proof. It is clear in this case that the set $E$ of Theorem 1.9 is $E_{1}$ and that $E_{0}=\operatorname{sp}\left(T_{0}\right)$. Thus, $\operatorname{sp}\left(T_{s}\right) \subset I_{s}\left(E_{0}, E_{1}\right)$.

Now suppose that $z \in I_{s}\left(E_{0}, E_{1}\right)$. Since the conclusion of the theorem is obvious in case $s=0$ or 1 , we assume $0<s<1$. To show that $z \in \operatorname{sp}\left(T_{s}\right)$ we first show that

$$
|x(z)| \leqq\|x\|_{B_{s}}, \quad x \in R\left(E_{1}\right) .
$$

Let $\epsilon>0$. Since $B_{0} \cap B_{1}=B_{1}$, it follows from [4, 2.5] that there is an $f$ in $\mathscr{F}=\mathscr{F}\left(B_{0}, B_{1}\right)$ (see [2] for definition of $\mathscr{F}$ ) such that $f(s)=x$,

$$
\|x\|_{B_{s}}+\epsilon \geqq\|f\|_{F}
$$

and $f(\xi)=\Sigma c_{j}(\xi) x_{j}$ where the sum is finite, each $x_{j} \in B_{1}$ and each $c_{j}$ is continuous on $0 \leqq \operatorname{Re} \xi \leqq 1$, vanishes at $\infty$ and is analytic on $0<\operatorname{Re} \xi<$ 1. It is clear from the definition of $C_{R}\left(E_{1}\right)$ that each $x_{j}$ can be replaced by a $y_{j}$ in $R\left(E_{1}\right)$ such that for the corresponding function $f_{1}(\xi)=\Sigma c_{j}(\xi) y_{j}$ we have

$$
\left\|f(\xi)-f_{1}(\xi)\right\|_{B_{1}}<\epsilon, \quad 0 \leqq \operatorname{Re} \xi \leqq 1
$$

From (3) and the fact that the norm of $B_{1}$ dominates the norm of $B_{0}$ on $B_{1}$ we have 


$$
\left\|f-f_{1}\right\|_{\mathscr{F}}<\epsilon
$$

Given a pair $(r, F)$ which satisfies (a) through (c) of 1.3 let $\mathscr{F}_{1}$ denote the space

$$
\mathscr{F}\left(C_{R}(L(1, F)), C_{R}(L(r, F))\right)
$$

From Theorem 2.6 and the fact that each of the functions $y_{j}$ (there are only a finite number of functions $\left.y_{j}\right)$ is in $R\left(E_{1}\right)$ it is clear that $(r, F)$ can be chosen so that $f_{1} \in \mathscr{F}_{1}$ and

$$
(1+\epsilon)\left\|f_{1}\right\|_{\mathscr{F}} \geqq\left\|f_{1}\right\|_{\Phi_{1}} .
$$

If we let $E_{k}$ in $[4,5.3]$ (see 2.1 above) be $L\left(r^{k}, F\right), k=0,1$ and define $\omega$ by: $\omega(z)=0, z \in E_{0}$ and $\omega(z)=\log |F(z)|$ for $z \in E_{1} \backslash E_{0}$; then we can conclude from $[4,5.3]$ that

$$
\left\|f_{1}\right\|_{\mathcal{F}_{1}} \geqq\left|f_{1}(z, s)\right|
$$

From Theorem 2.6 it is clear that $E_{1} \supset I_{s}\left(E_{0}, E_{1}\right)$. In particular, $z \in$ $E_{1}$. From this and (3) we obtain

$$
\left|x(z)-f_{1}(z, s)\right| \leqq\left\|x-f_{1}(s)\right\|_{B_{1}}<\epsilon .
$$

Since $\epsilon$ is arbitrary, (1) now follows by combining (2), (4), (5), (6) and (7).

Now suppose that $x \in R\left(E_{1}\right)$ such that $x(z) \neq 0$. From (1) we obtain

$$
|x(z)|=\left|x(z)-\left(z-T_{s}\right) y(z)\right| \leqq\left\|x-\left(z-T_{s}\right) y\right\|_{B_{s}}, \quad y \in R\left(E_{1}\right) .
$$

Since $R\left(E_{1}\right)$ is dense in $B_{s}$, this last inequality shows that the range of $\left(z-T_{s}\right)$ is $\neq B_{s}$. Thus, $x \in \operatorname{sp}\left(T_{s}\right)$, which completes the proof.

4. A criterion for constant spectra. This section is devoted to Theorem 4.1, which gives a criterion for $\operatorname{sp}\left(T_{s}\right)=\operatorname{sp}\left(T_{0}\right)$, $0 \leqq s<1$.

THEOREM 4.1. Suppose that $T_{i}$ is a bounded linear operator on $B^{\prime}, j=0,1$, that $T_{0}$ and $T_{1}$ agree on $B_{0} \cap B_{1}$ and that to each $\epsilon>0$ there correspond subspaces $Q_{1, j}, \cdots, Q_{n, j}$ of $B_{j}, j=0,1$ with the following properties: (a) each space $Q_{k, j}$ is $T_{j}$ invariant ; (b) $B_{j}$ is the direct sum of the spaces $Q_{k, j}, j=0,1$; (c) $Q_{k, 0} \cap Q_{k, 1}$ is dense in both $Q_{k, 0}$ and $Q_{k, 1}$ for all $j$; (d) $\operatorname{sp}\left(T_{0} \mid Q_{k, 0}\right) \subset \operatorname{sp}\left(T_{1} \mid Q_{k, 1}\right)$ for each $k ;(\mathrm{e}) \operatorname{sp}\left(T_{0} \mid Q_{k, 0}\right)$ is contained in $D(z, \epsilon)$ for some $z$ in $\operatorname{sp}\left(T_{0}\right)$ depending on $k, k=1,2, \cdots, n$. Then 


$$
\operatorname{sp}\left(T_{s}\right)=\operatorname{sp}\left(T_{0}\right) \quad \text { for } \quad 0 \leqq s<1
$$

Proof. Since $\operatorname{sp}\left(a T_{s}\right)=\operatorname{sp}\left(T_{s}\right)$ for any complex number $a$, it clearly suffices to prove the theorem assuming $\left\|T_{0}\right\| \leqq 1 / 2$ and $\left\|T_{1}\right\| \leqq 1 / 2$. Fix $s$ and let $\epsilon>0$. Choose $\delta$ corresponding to $\epsilon, s$ and $K=$ $\operatorname{sp}\left(T_{0}\right)\left(\operatorname{sp}\left(T_{0}\right) \subset \mathrm{D}(0,1 / 2]\right)$ in Lemma 1.5 . Let $Q_{k, j}$ be the spaces corresponding to $\delta$ as in the theorem. Let $U_{k, j}$ be the restriction of the operator $T_{j}$ to the space $Q_{k, j}$. Let $Q_{k, s}$ denote the space $\left[Q_{k, 0}, Q_{k, 1}\right]_{s}$, where $Q_{k, 0}$ and $Q_{k, 1}$ are regarded as embedded in $V$ (see 1.1) in the natural manner. If $x \in Q_{k, 0} \cap Q_{k, 1}$, then $T_{s} x=T_{0} x=T_{1} x$; consequently, $T_{s} x \in Q_{k, 0} \cap Q_{k, 1}$. This shows that $Q_{k, s}$ is an invariant subspace of $T_{s}$. Let $U_{k, s}$ denote the restriction of $T_{s}$ to $Q_{k, s \cdot}$. For each $k$ it is clear from (d) that the hypotheses of Theorem 1.9 are satisfied in the case of the operators $U_{k, l}, j=0,1$; thus, we conclude that

$$
\operatorname{sp}\left(U_{k, s}\right) \subset I_{s}\left(\operatorname{sp}\left(U_{k, 0}\right), E_{k}\right)
$$

where $E_{k}$ is the set corresponding to $U_{k, j}$ as in Theorem 1.9. Because of the choice of subspaces $Q_{k, j}$ we have $\operatorname{sp}\left(U_{k, 0}\right) \subset D\left(z_{k}, \delta\right)$ for some $z_{k} \in$ $\operatorname{sp}\left(T_{0}\right)$. Since $\left\|T_{k}\right\| \leqq 1 / 2, \operatorname{sp}\left(T_{k}\right) \subset D(0,1] ;$ this together with Theorem 1.7 implies that $E_{k} \subset D(0,1]$. From these observations, Lemma 1.4, Lemma 1.5 and the way in which $\delta$ was chosen it follows that

$$
\operatorname{sp}\left(U_{k, s}\right) \subset \operatorname{sp}\left(T_{0}\right)+D(0, \epsilon) .
$$

Since $\operatorname{sp}\left(T_{s}\right)=\cup \operatorname{sp}\left(U_{k, s}\right), k=1,2, \cdots, n$, it follows that

$$
\operatorname{sp}\left(T_{s}\right) \subset \operatorname{sp}\left(T_{0}\right)+D(0, \epsilon) .
$$

The conclusion of the theorem follows from this and the fact that $\epsilon$ was chosen arbitrarily.

REMARK. A special case of Theorem 4.1 can be proved by slightly extending a method used by Hirschman [3] for determining the spectrum of certain multipliers. The special case occurs if, in addition to the assumptions in Theorem 4.1, we assume that each operator $U_{k}$, which is obtained by restricting $T_{0}$ to $Q_{k, 0}$, has the property that the norm of $\beta I-U_{k}$ is equal to its spectral radius for each complex number $\beta$. Suppose $\lambda \notin \operatorname{sp}\left(T_{0}\right)$. Let $d$ be the distance from $\lambda$ to $\operatorname{sp}\left(T_{0}\right)$. Let $0<s<1$ and choose $\epsilon$ so that $\epsilon^{1-s}\left(2\left\|T_{1}\right\| / d\right)^{s} \leqq 1 / 2$. Choose the subspaces $Q_{k, j}$ corresponding to $\epsilon$. Fix $k$ and let $S_{j}$ be the operator obtained by restricting $T_{j}$ to $Q_{k, j}$ and let $S_{s}$ be the corresponding operators on the interpolation spaces. Let $\beta \in \operatorname{sp}\left(S_{0}\right)$. Then $\lambda I-S_{s}=$ $(\lambda-\beta) I+\beta I-S_{s}=(\lambda-\beta)\left(I-A_{s}\right)$ where, in general, $A_{t}=$ 
$(\beta-\lambda)^{-1}\left(\beta I-S_{t}\right)$. Now $\left\|A_{s}\right\|_{s} \leqq\left\|A_{0}\right\|^{1-s}\left\|A_{1}\right\|^{s} \leqq 1 / 2$. Thus, $\lambda I-S_{s}$ has an inverse. Since $k$ was arbitrary, we see that $\lambda \notin \operatorname{sp}\left(T_{s}\right)$.

\section{REFERENCES}

1. Lars V. Ahlfors, Complex Analysis, 2nd Ed., McGraw-Hill Book Co., New York, 1966.

2. A. P. Calderon, Intermediate spaces and interpolation, the complex method, Studia Math., 24 (1964), 113-190.

3. I. I. Hirshman, On multiplier transformations, Duke Math. J., 26 (1959), 221-242.

4. James D. Stafney, The spectrum of an operator on an interpolation space, Trans. Amer. Math. Soc., V144 (1969), 333-350.

5. M. Tsuji, Potential theory in modern function theory, Maruzen Co., Ltd., Tokio, 1959.

Received January 14, 1974.

UNIVERSITY OF CALIFORNIA, RIVERSIDE 



\section{PACIFIC JOURNAL OF MATHEMATICS}

\section{EDITORS}

RICHARD ARENS (Managing Editor)

University of California

Los Angeles, California 90024

\author{
R. A. Beaumont \\ University of Washington \\ Seattle, Washington 98105
}

\section{J. DugundII}

Department of Mathematics

University of Southern California

Los Angeles, California 90007

D. Gilbarg and J. Milgram

Stanford University

Stanford, California 94305

\section{ASSOCIATE EDITORS}
E. F. BECKENBACH
B. H. NeumanN
F. WoLF
K. YoshidA

\section{SUPPORTING INSTITUTIONS}

\author{
UNIVERSITY OF BRITISH COLUMBIA \\ CALIFORNIA INSTITUTE OF TECHNOLOGY \\ UNIVERSITY OF CALIFORNIA \\ MONTANA STATE UNIVERSITY \\ UNIVERSITY OF NEVADA \\ NEW MEXICO STATE UNIVERSITY \\ OREGON STATE UNIVERSITY \\ UNIVERSITY OF OREGON \\ OSAKA UNIVERSITY
}

\author{
UNIVERSITY OF SOUTHERN CALIFORNIA \\ STANFORD UNIVERSITY \\ UNIVERSITY OF TOKYO \\ UNIVERSITY OF UTAH \\ WASHINGTON STATE UNIVERSITY \\ UNIVERSITY OF WASHINGTON \\ AMERICAN MATHEMATICAL SOCIETY
}

The Supporting Institutions listed above contribute to the cost of publication of this Journal, but they are not owners or publishers and have no responsibility for its contents or policies.

Mathematical papers intended for publication in the Pacific Journal of Mathematics should be in typed form or offset-reproduced (not dittoed), double spaced with large margins. Underline Greek letters in red, German in green, and script in blue. The first paragraph or two must be capable of being used separately as a synopsis of the entire paper. Items of the bibliography should not be cited there unless absolutely necessary, in which case they must be identified by author and Journal, rather than by item number. Manuscripts, in duplicate, may be sent to any one of the four editors. Please classify according to the scheme of Math. Reviews, Index to Vol. 39. All other communications should be addressed to the managing editor, or Elaine Barth, University of California, Los Angeles, California, 90024.

100 reprints are provided free for each article, only if page charges have been substantially paid. Additional copies may be obtained at cost in multiples of 50.

The Pacific Journal of Mathematics is issued monthly as of January 1966. Regular subscription rate: $\$ 72.00$ a year (6 Vols., 12 issues). Special rate: $\$ 36.00$ a year to individual members of supporting institutions.

Subscriptions, orders for back numbers, and changes of address should be sent to Pacific Journal of Mathematics, 103 Highland Boulevard, Berkeley, California, 94708.

PUBLISHED BY PACIFIC JOURNAL OF MATHEMATICS, A NON-PROFIT CORPORATION Printed at Jerusalem Academic Press, POB 2390, Jerusalem, Israel.

\section{Copyright (C) 1975 Pacific Journal of Mathematics All Rights Reserved}




\section{Pacific Journal of Mathematics

Vol. 60, No. $2 \quad$ October, 1975

Waleed A. Al-Salam and A. Verma, A fractional Leibniz q-formula ........... 1

Robert A. Bekes, Algebraically irreducible representations of $L_{1}(G) \ldots \ldots \ldots \ldots 11$

Thomas Theodore Bowman, Construction functors for topological

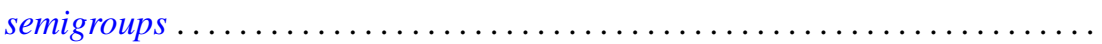

Stephen LaVern Campbell, Operator-valued inner functions analytic on the

closed disc. II .........................................

Leonard Eliezer Dor and Edward Wilfred Odell, Jr., Monotone bases in $L_{p} \ldots \ldots$.

Yukiyoshi Ebihara, Mitsuhiro Nakao and Tokumori Nanbu, On the existence of

global classical solution of initial-boundary value problem for

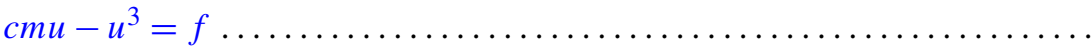

Y. Gordon, Unconditional Schauder decompositions of normed ideals of

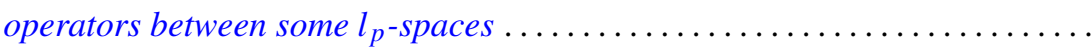

Gary Grefsrud, Oscillatory properties of solutions of certain nth order functional

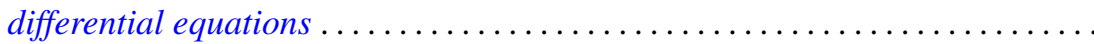

Irvin Roy Hentzel, Generalized right alternative rings ...................

Zensiro Goseki and Thomas Benny Rushing, Embeddings of shape classes of compacta in the trivial range .................................

Emil Grosswald, Brownian motion and sets of multiplicity . .

Donald LaTorre, A construction of the idempotent-separating congruences on a

bisimple orthodox semigroup .

Pjek-Hwee Lee, On subrings of rings with involution ...

Marvin David Marcus and H. Minc, On two theorems of Frobenius ...

Michael Douglas Miller, On the lattice of normal subgroups of a direct

product. .

Grattan Patrick Murphy, A metric basis characterization of Euclidean space

Roy Martin Rakestraw, A representation theorem for real convex functions ....

Louis Jackson Ratliff, Jr., On Rees localities and $H_{i}$-local rings ...

Simeon Reich, Fixed point iterations of nonexpansive mapping . .

Domenico Rosa, $B$-complete and $B_{r}$-complete topological algebras ...

Walter Roth, Uniform approximation by elements of a cone of real-valued

functions ....

Helmut R. Salzmann, Homogene kompakte projektive Ebenen

Jerrold Norman Siegel, On a space between $B H$ and $B_{\infty} \ldots$

235

Robert C. Sine, On local uniform mean convergence for Markov operators

James D. Stafney, Set approximation by lemniscates and the spectrum of an

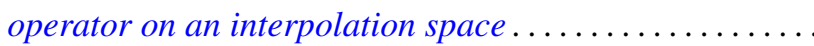

Árpád Száz, Convolution multipliers and distributions .......

Kalathoor Varadarajan, Span and stably trivial bundles ..........

Robert Breckenridge Warfield, Jr., Countably generated modules over

commutative Artinian rings....................... 